\title{
Loss of Human Leukocyte Antigen Class I Expression Is Associated with Poor Prognosis in Patients with Advanced Breast Cancer
}

\author{
Hong Sik Park · Uiju Cho \\ So Young Im • Chang Young Yoo \\ Ji Han Jung · Young Jin Suh ${ }^{1}$ \\ Hyun Joo Choi
}

Departments of Hospital Pathology and ' 1 Surgery, St. Vincent's Hospital, College of Medicine, The Catholic University of Korea, Seoul, Korea

Received: August 9, 2018

Revised: September 21, 2018

Accepted: October 11, 2018

\section{Corresponding Author}

Hyun Joo Choi, MD

Department of Hospital Pathology, St. Vincent's

Hospital, College of Medicine, The Catholic

University of Korea, 93 Jungbu-daero, Paldal-gu,

Suwon 16247 , Korea

Tel: +82-31-249-7592

Fax: +82-31-244-6786

E-mail: chj0103@catholic.ac.kr

\begin{abstract}
Background: Human leukocyte antigen class I (HLA-I) molecules play important roles in regulating immune responses. Loss or reduction of HLA-I expression has been shown to be associated with prognosis in several cancers. Regulatory T-cells (Tregs) also play critical functions in immune response regulation. Evaluation of HLA-I expression status by the EMR8-5 antibody and its clinical impact in breast cancer have not been well studied, and its relationship with Tregs remains unclear. Methods: We evaluated HLA-I expression and Treg infiltration by immunohistochemistry in 465 surgically resected breast cancer samples. We examined the correlation between HLA-I expression and Treg infiltration and clinicopathologic characteristics and survival analyses were performed. Results: Total loss of HLA-I expression was found in 84 breast cancer samples (18.1\%). Univariate survival analysis revealed that loss of HLA-I expression was significantly associated with worse disease-specific survival (DSS) ( $p=.029$ ). HLA-I was not an independent prognostic factor in the entire patient group, but it was an adverse independent prognostic factor for DSS in patients with advanced disease (stage II-IV) $(p=.031)$. Treg numbers were significantly higher in the intratumoral stroma of HLA-I-positive tumors than in HLA-I-negative tumors (median 6.3 cells/high power field vs 2.1 cells/high power field, $p<.001$ ). However, Tregs were not an independent prognostic factor in our cohort. Conclusions: Our findings suggest that the loss of HLA-I expression is associated with poor prognosis in breast cancer patients, highlighting the role of $\mathrm{HLA}-\mathrm{I}$ alterations in immune evasion mechanisms of breast cancer. HLA-I could be a promising marker that enables the application of more effective and precise immunotherapies for patients with advanced breast cancer.
\end{abstract}

Key Words: Breast neoplasms; HLA antigens; Major histocompatibility complex; Lymphocytes, tumor-infiltrating; T-lymphocytes, regulatory
Host immune systems can recognize and eliminate cells presenting abnormal tumor antigens, such as those against mutated oncoproteins. However, genomic instability and cancer-promoting inflammation can accelerate the acquisition of genetic and epigenetic alterations that allow cancer cells to evade the innate and adaptive immune systems. ${ }^{1}$ One alteration that helps cancer cells escape from cytotoxic T lymphocyte recognition is the down-regulation or complete loss of human leukocyte antigen class I (HLA-I) expression, which is induced by changes in HLA-A, $-\mathrm{B}$, and $-\mathrm{C}$ variants and the $\beta-2$-microglobulin chain. ${ }^{2}$ Aberrant expression of HLA-I on cancer cells has been frequently observed in cancers of various histological types and is associated with clinical outcome. ${ }^{3}$ Aberrant expression of HLAI ranges from loss of a single allele to complete loss of HLA-I expression. ${ }^{4}$ Down-regulation of HLA-I expression has also been observed in breast cancer ${ }^{2,3,5,6}$ and was reported in up to $85 \%$ of the primary tumors. The destruction of HLA-I-positive can- cer cells by a specific T cell-mediated immune reaction, "T-cell immune selection," is thought to underlie HLA-1 down-regulation in breast cancer. ${ }^{7}$

Few studies have examined the clinical implications of HLAI expression in breast cancer, and the results have been conflicting in different subsets. ${ }^{5,8,9}$ Among these analyses, even fewer studies used the recently developed anti-pan HLA-I monoclonal antibody (EMR8-5), which has shown improved suitability for immunostaining formalin-fixed paraffin-embedded (FFPE) tissue. ${ }^{6,9-12}$

Regulatory T cells (Tregs) are a subset of helper T lymphocytes that play an important role in tumor-induced tolerance to immune surveillance. ${ }^{13}$ Tregs were found to be significantly increased in the tumor stroma of several cancer types and act as immune suppressors. ${ }^{14,15}$ Tregs were initially characterized as CD4- and CD25expressing cells. Further investigation demonstrated that Tregs express forkhead box protein P3 (FOXP3) and hold essential role in their development and function. ${ }^{16}$ Tregs can be specifically 
detected in tissue sections by FOXP3 staining. Several studies showed that an increased number of intratumoral Tregs was associated with poor clinical outcome in breast cancer, ${ }^{8,17}$ while other investigations found no prognostic significance. ${ }^{16}$ Therefore, its prognostic value still remains controversial. Furthermore, few studies have examined Treg number and HLA-I expression in breast cancer.

Here we examined HLA-I expression in primary invasive breast cancer and some matched metastatic breast cancer tissues using the anti-pan HLA-I antibody EMR8-5 and investigated the possible relationship between Treg infiltration and HLA-I expression in tumors. We also explored the association between HLA-I expression with clinicopathological factors and the clinical implications of HLA-I loss in breast cancer.

\section{MATERIALS AND METHODS}

\section{Patients and tissue samples}

We collected 465 cases of invasive breast cancer from the archives of St. Vincent's Hospital, Suwon between January 2003 and December 2011. Among them, 18 cases had paired tissues of metastatic breast cancer that developed after the initial surgery. All patients underwent surgical resection and were treated according to standard treatment guidelines, as outlined during that timeframe, regarding chemotherapy and radiotherapy. Data regarding patient demographics, clinicopathological parameters and survival were retrospectively collected from hospital medical records. Pathologic stages were categorized according to the seventh edition of the TNM classification by the American Joint Committee on Cancer. ${ }^{18}$ According to the clinical characteristics, tumors in stage I were defined as early cases and those in stages II, III and IV were defined as advanced cases. All samples and medical record data were anonymized before use in this study. Formal written informed consent was not required based on a waiver by the Institutional Review Board (IRB) of St. Vincent's Hospital. The use of medical record data and tissue samples for this study was approved by the IRB (VC16SISI0214).

\section{Construction of tissue microarray}

We constructed tissue microarrays (TMAs) from 465 FFPE breast cancer blocks. A morphologically representative tumor area in each of the donor blocks was selected based on the hematoxylin and eosin (H\&E)-stained sections and the site corresponding to the confirmed tumor site in the paraffin block was marked. The selected area was harvested using a 2-mm Quick-Ray tissue microarrayer device (Micro Digital Co., Seoul, Korea) and then trans- ferred to a recipient TMA mold. One core per case was used for the construction of the TMA. One slide from each of the TMA blocks was stained with H\&E to confirm the presence of tumor tissue.

\section{Immunohistochemistry and evaluation of immunohistochemical staining}

The TMA blocks were cut into 4 - $\mu \mathrm{m}$-thick sections and mounted on precoated glass slides. Briefly, TMA sections were dried, deparaffinized and rehydrated following standard procedures. Immunohistochemistry (IHC) for HLA-I (HLA-ABC, clone EMR8-5, mouse monoclonal, 1:200 dilution, Abcam, Cambridge, MA, USA) and FOXP3 (clone 236A/E7, mouse monoclonal, 1:100 dilution, Abcam) was carried out using a Ventana NX automated IHC system (Ventana Medical Systems, Tucson, AZ, USA). Heat-induced antigen retrieval was performed using cell conditioning solution (CC1, a proprietary buffer). Normal tonsil tissues served as a positive control for all the antibodies. HLA-I expression was categorized as "negative" when there was a complete absence of HLA-I expression in the tumor cells and as "positive" when HLA-I expression was observed in any of the tumor cell membranes. HLA-I expression was also assessed according to the percentage of HLA-I positive cells in a given field: low $(<25 \%)$, intermediate $(25 \%-75 \%)$ or high $(>75 \%)$ expression. Tregs were identified by FOXP3 staining. Lymphocytes with nuclear FOXP3 expression regardless of the intensity were counted as FOXP3-positive cells. The number of FOXP3positive $\mathrm{T}$ cells was counted at the entire peritumoral area in five high power fields (HPF) per case, and the average number of cells per a HPF was calculated. Treg infiltration was categorized as "absent" when there were no FOXP3-positive T cells and as "present" when more than one FOXP3-positive T cell was observed. All slides were evaluated by two pathologists blinded to patient information.

A combination of HLA-I expression status and Treg infiltration status yielded four immunologic groups (group 1, HLA- $-^{\text {negative }}$ / Treg absent; group 2, HLA-- ${ }^{\text {negative }} /$ Treg $^{\text {present; }}$; group 3, HLA-I Positive/ $^{\text {and }}$ Treg ${ }^{\text {absent }}$; and group 4, HLA-I $\mathrm{I}^{\text {positive }} /$ Treg $^{\text {present }}$ ) and they were analyzed for clinical significance.

\section{Definition of breast cancer subtypes}

Expressions of standard biomarkers including estrogen receptor (ER), progesterone receptor (PR), human epidermal growth factor receptor 2 (HER2), and Ki67 were evaluated from whole sections at the time of diagnosis and the results were obtained from the pathology reports. For ER and PR, an Allred method 
was used for scoring and a score $\geq 3$ was considered positive. ${ }^{19}$ For HER2, a score of 3+ on IHC or the presence of gene amplification on in situ hybridization was considered positive for amplification. Each of the intrinsic breast cancer subtypes was classified as follows: luminal A type (ER + and/or PR +, HER2-, Ki67 $<14 \%$,); luminal B type (ER + and/or PR +, HER2-, Ki67 $\geq 14 \%$ or ER + and/or PR +, HER $2+$ ); HER2-positive type (ER-/ $\mathrm{PR}-/ \mathrm{HER} 2+$ ); and triple-negative type (ER-/PR-/HER2-).

\section{Statistical analysis}

The chi-square test or Fisher exact test was used to analyze the correlation between HLA-I expression and clinicopathological parameters and Student's test was used to analyze the differences in mean values. Disease-specific survival (DSS) time was measured from the time of initial diagnosis until death specifically caused by breast cancer or until the end of follow-up. Disease-free survival (DFS) time was measured from the time of initial diagnosis until disease recurrence, progression or metastasis. Survival data were analyzed using Kaplan-Meier survival curves and the differences between curves were analyzed using log-rank tests. Multivariate analysis for the DSS and DFS were performed using the Cox proportional hazards model. Two-tailed p-values of $<.05$ were considered significant. Analysis and data graphing listed above were performed using SPSS ver. 21.0 (IBM Corp., Armonk, NY, USA).

\section{RESULTS}

HLA-I expression and Treg infiltration in primary tumors and their correlation with clinicopathologic factors in breast cancer patients

A total of 465 cases of invasive breast cancer were included in this study. Patients and their clinicopathological characteristics are described in Table 1. The median age of patients was 53.2 years (standard deviation, 11.8; range, 30 to 86 years). The median follow-up of patients was 62 months (range, 1 to 172 months), and 32 patients $(6.9 \%)$ died from breast cancer. A total of 63 patients $(13.5 \%)$ had disease progression, recurrence or metastasis after the initial diagnosis.

HLA-I expression in primary tumor cells was positive in 381 patients (81.9\%) and negative in 84 patients (18.1\%) (Fig. 1). In the 381 patients with positive HLA-I expression, 101 patients $(26.5 \%)$ had low expression (<25\%), 74 patients (19.4\%) had mid expression (25\%-75\%) and 206 patients (54\%) had high expression (> 75\%). HLA-I expression was uniformly strong in stromal cells of all the cases.
Infiltration of Tregs was generally absent or low in all 465 tumors. The median number of tumor-infiltrating Tregs was 1.8 cells/HPF (range, 0 to 91), and 123 cases (26.5\%) had no Tregs.

We analyzed the correlation between HLA-I expression and Treg presence with clinicopathological features (Table 1). Loss of HLA-I expression was associated with old age, low histologic grade, low nuclear grade and luminal A intrinsic type (all $\mathrm{p}<$ .05). Presence of Treg infiltration was significantly correlated with tumors with high histologic grade, high nuclear grade, negative hormone receptor (HR) status and triple negative intrinsic type $($ all $\mathrm{p}<.05)$.

\section{HLA-I expression and Treg infiltration in metastatic tumors}

Of the 63 patients who developed progression, recurrence or metastasis after the primary surgery, paired metastatic tumor materials were available for 18 patients and these tissues were evaluated for HLA-I expression. Notably, patients were not treated with immunotherapy during the course of metastatic disease. In the metastatic subset, HLA-I expression was positive in 16 patients $(89 \%)$ and negative in two (11\%). All HLA-I positive cases showed strong expression on tumor cell membranes. Loss of HLA-I expression occurred more frequently in the primary tumor than the metastatic tumor $(18.1 \%$ vs $11 \%$, respectively), but this difference was not statistically significant ( $\mathrm{p}=$ .441). There was little change in HLA-I expression between the paired primary and metastatic lesions. Only one case with loss of HLA-I expression in the primary lesion showed HLA-I expression in the metastatic lesion.

We also examined Tregs in the metastatic subset. The median number of infiltrating Tregs was 2 cells/HPF (range, 0 to 25.8) and four cases (22\%) had no Tregs in the tumor microenvironment. There was no significant difference in the number of Tregs between primary and metastatic tumors $(\mathrm{p}=.641)$.

\section{Correlation of HLA-I expression and Treg infiltration}

We evaluated the correlation between HLA-I expression and Treg infiltration in primary tumors and metastatic tumors. In the primary tumors, HLA-I-negative tumors had a significantly lower number of Tregs in their tumor microenvironment than HLA-I-positive tumors (median, 2.1 cells/HPF vs 6.3 cells/ HPF; $\mathrm{p}<.001$ ) (Fig. 2). Likewise, when cases were categorized into absent and present Treg infiltration, the loss of HLA-I expression was significantly correlated with the absence of Treg infiltration (Table 1). 
Table 1. Baseline characteristics of the patients according to the HLA-I expression and regulatory T-cell infiltration

\begin{tabular}{|c|c|c|c|c|c|c|c|}
\hline \multirow{2}{*}{ Characteristic } & \multirow{2}{*}{ Total $(n=465)$} & \multicolumn{2}{|c|}{ HLA-I } & \multirow{2}{*}{$p$-value } & \multicolumn{2}{|c|}{ Regulatory T cell } & \multirow{2}{*}{$p$-value } \\
\hline & & Negative $(n=84)$ & Positive $(n=381)$ & & Absent $(n=123)$ & Present $(n=342)$ & \\
\hline Age at diagnosis $(y r)^{a}$ & $53.2 \pm 11.8$ & $55.7 \pm 12.0$ & $52.6 \pm 11.8$ & .030 & $54.4 \pm 12.3$ & $52.7 \pm 11.7$ & .190 \\
\hline No. of Tregs (cells/HPF) & $5.5 \pm 10.0$ & $2.1 \pm 4.5$ & $6.3 \pm 10.7$ & $<.001$ & - & - & - \\
\hline \multicolumn{8}{|l|}{ Sex } \\
\hline Female & $464(99.8)$ & $84(100)$ & $380(99.7)$ & $.999^{b}$ & $122(99.2)$ & $342(100)$ & $.265^{b}$ \\
\hline Male & $1(0.2)$ & 0 & $1(0.3)$ & & $1(0.8)$ & 0 & \\
\hline \multicolumn{8}{|l|}{ Operation } \\
\hline Lumpectomy & 294 (63.2) & $46(54.8)$ & $248(65.1)$ & .081 & $76(61.8)$ & $218(63.7)$ & .700 \\
\hline Mastectomyc & $171(36.8)$ & $38(45.2)$ & $133(34.9)$ & & 47 (38.2) & $124(36.3)$ & \\
\hline \multicolumn{8}{|l|}{ Multiplicity } \\
\hline Solitary tumor & 407 (87.5) & $73(86.9)$ & $334(87.7)$ & .856 & $110(89.4)$ & $296(86.8)$ & .456 \\
\hline Multiple tumor & $58(12.5)$ & $11(13.1)$ & $47(12.3)$ & & $13(10.6)$ & $45(13.2)$ & \\
\hline \multicolumn{8}{|l|}{ Histologic grade } \\
\hline Low (grade 1) & $123(26.5)$ & $36(42.9)$ & 87 (22.8) & $<.001$ & $53(43.1)$ & $70(20.5)$ & $<.001$ \\
\hline High (grade 2, 3) & $342(73.5)$ & $48(57.1)$ & $294(77.2)$ & & $70(56.9)$ & $272(79.5)$ & \\
\hline \multicolumn{8}{|l|}{ Nuclear grade } \\
\hline Low (grade 1) & $43(9.2)$ & $17(20.2)$ & $26(6.8)$ & $<.001$ & $19(15.4)$ & $24(7.0)$ & $<.001$ \\
\hline High (grade 2, 3) & $422(90.8)$ & 67 (79.8) & $355(93.2)$ & & $104(84.6)$ & $318(93.0)$ & \\
\hline \multicolumn{8}{|l|}{ Lymphovascular invasion } \\
\hline Absent & $342(73.5)$ & 66 (78.6) & $276(72.4)$ & .249 & $91(74.0)$ & $251(73.4)$ & .898 \\
\hline Present & $123(26.5)$ & $18(21.4)$ & $105(27.6)$ & & $32(26.0)$ & $91(26.6)$ & \\
\hline \multicolumn{8}{|l|}{ Perineural invasion } \\
\hline Absent & $424(91.2)$ & 78 (92.9) & $346(90.8)$ & .550 & $110(89.4)$ & $314(91.8)$ & .424 \\
\hline Present & $41(8.8)$ & $6(7.1)$ & $35(9.2)$ & & $18(10.6)$ & $28(8.2)$ & \\
\hline \multicolumn{8}{|l|}{ T category } \\
\hline $\mathrm{T} 1$ & $245(52.7)$ & $50(59.5)$ & $195(51.2)$ & .544 & $72(58.5)$ & $172(50.3)$ & .110 \\
\hline $\mathrm{T} 2$ & $200(43.0)$ & $30(35.7)$ & $170(44.6)$ & & 47 (38.2) & $154(45.0)$ & \\
\hline T3 & $19(4.1)$ & $4(4.8)$ & $15(3.9)$ & & $3(2.4)$ & $16(4.11)$ & \\
\hline $\mathrm{T} 4$ & $1(0.2)$ & 0 & $1(0.3)$ & & $1(0.8)$ & 0 & \\
\hline \multicolumn{8}{|l|}{ N category } \\
\hline NO & $313(67.3)$ & $59(70.2)$ & $254(66.7)$ & .610 & $81(65.9)$ & $232(67.8)$ & .787 \\
\hline $\mathrm{N} 1$ & $106(22.8)$ & $15(17.9)$ & $91(23.9)$ & & $28(22.8)$ & 78 (22.8) & \\
\hline N2 & $30(6.5)$ & $6(7.1)$ & $24(6.3)$ & & $8(6.5)$ & 22 (73.3) & \\
\hline N3 & $16(3.4)$ & $6(4.8)$ & $12(3.1)$ & & & & \\
\hline \multicolumn{8}{|l|}{ Lymph node metastasis } \\
\hline Absent & $313(67.3)$ & 59 (70.2) & $254(66.7)$ & .528 & $81(65.9)$ & $232(67.8)$ & .688 \\
\hline Present & $152(32.7)$ & $25(29.8)$ & $127(33.3)$ & & $42(34.1)$ & $110(32.2)$ & \\
\hline \multicolumn{8}{|l|}{ Distant metastasis } \\
\hline Absent & $453(97.4)$ & 80 (95.2) & $373(97.9)$ & .164 & $119(96.7)$ & $334(97.7)$ & $.396^{c}$ \\
\hline Present & $12(2.6)$ & $4(4.8)$ & $8(2.1)$ & & $4(3.3)$ & $8(2.3)$ & \\
\hline \multicolumn{8}{|l|}{ AJCC stage } \\
\hline I & $188(40.4)$ & $41(48.8)$ & $147(38.6)$ & .163 & $53(43.1)$ & $135(39.5)$ & .849 \\
\hline$\|$ & $220(47.3)$ & 31 (36.9) & $189(49.6)$ & & $54(43.7)$ & $166(48.5)$ & \\
\hline III & $47(10.1)$ & $9(10.7)$ & $38(10.0)$ & & $13(10.6)$ & $34(9.95)$ & \\
\hline IV & $10(2.2)$ & $3(3.6)$ & $7(1.8)$ & & $3(2.4)$ & $7(2.0)$ & \\
\hline ER status & & & & & & & \\
\hline Negative & $182(39.1)$ & 33 (39.3) & $149(39.1)$ & .976 & 35 (28.5) & $147(43.0)$ & .005 \\
\hline Positive & $283(60.9)$ & $51(60.7)$ & $232(60.9)$ & & $88(71.5)$ & $195(57.0)$ & \\
\hline PR status & & & & & & & \\
\hline Negative & $213(45.8)$ & $37(44.0)$ & $176(46.2)$ & .721 & 45 (36.6) & $168(49.1)$ & .017 \\
\hline Positive & $252(54.2)$ & $47(56.0)$ & $205(53.8)$ & & $78(63.4)$ & $174(50.9)$ & \\
\hline HER2 status & & & & & & & \\
\hline Negative & $333(71.6)$ & 64 (76.2) & 269 (70.6) & .304 & 94 (76.4) & 239 (69.9) & .168 \\
\hline Positive & $132(28.4)$ & $20(23.8)$ & $112(29.4)$ & & 29 (23.6) & $103(30.1)$ & \\
\hline
\end{tabular}


Table 1. Continued

\begin{tabular}{|c|c|c|c|c|c|c|c|}
\hline \multirow{2}{*}{ Characteristic } & \multirow{2}{*}{ Total $(n=465)$} & \multicolumn{2}{|c|}{ HLA-I } & \multirow{2}{*}{ p-value } & \multicolumn{2}{|c|}{ Regulatory T cell } & \multirow{2}{*}{$p$-value } \\
\hline & & Negative $(n=84)$ & Positive $(n=381)$ & & Absent $(n=123)$ & Present $(n=342)$ & \\
\hline \multicolumn{8}{|l|}{ Intrinsic subtype } \\
\hline Luminal A & $194(41.7)$ & $46(54.8)$ & $148(38.8)$ & .014 & $69(56.1)$ & $125(36.5)$ & .001 \\
\hline Luminal B & $116(24.9)$ & $11(9.5)$ & $105(27.6)$ & & $25(20.3)$ & $91(26.6)$ & \\
\hline HER2 & $70(15.1)$ & $14(16.7)$ & $56(14.7)$ & & $16(13.0)$ & $54(15.8)$ & \\
\hline Triple negative & 85 (18.3) & $13(15.5)$ & $72(18.9)$ & & $13(10.6)$ & $72(21.1)$ & \\
\hline \multicolumn{8}{|l|}{ Hormone receptor } \\
\hline Negative & $155(33.3)$ & $27(32.1)$ & $128(33.6)$ & .798 & $29(23.6)$ & $126(36.8)$ & .007 \\
\hline Positive & $310(66.7)$ & $57(67.9)$ & $253(66.4)$ & & $94(76.4)$ & $216(63.2)$ & \\
\hline \multicolumn{8}{|l|}{ Treatment } \\
\hline Neo-adjuvant chemotherapy & $16(3.4)$ & $82(97.6)$ & 367 (96.3) & $.748^{b}$ & $116(94.3)$ & $333(97.4)$ & $.099^{b}$ \\
\hline Surgery alone & $449(96.6)$ & $2(2.4)$ & $14(3.7)$ & & $7(5.7)$ & $9(2.6)$ & \\
\hline \multicolumn{8}{|l|}{ Regulatory $T$ cell } \\
\hline Absent & $123(26.5)$ & $37(44.0)$ & 86 (22.6) & $<.001$ & - & - & \\
\hline Present & 342 (73.5) & $47(56.0)$ & 295 (77.4) & & - & - & \\
\hline
\end{tabular}

Values are presented as mean \pm standard deviation or number (\%).

HLA-I, human leukocyte antigen class I; Treg, regulatory T cell; HPF, high-power field; AJCC, American Joint Committee on Cancer; ER, estrogen receptor; $\mathrm{PR}$, progesteron receptor; HER2, human epidermal growth factor receptor 2.

aStudent's $t$ test was used for comparison between the two group; 'Fisher exact test was used for comparison of between the two groups; 'This variable includes modified radical mastectomy and radical mastectomy.

\section{Prognostic significance of HLA-I expression and Treg infiltration in the total patient group}

Univariate analysis demonstrated that lymphovascular invasion $(\mathrm{p}=.001)$, perineural invasion $(\mathrm{p}=.002)$, distant metastasis $(\mathrm{p}<.001), \mathrm{T}$ category $(\mathrm{p}<.001), \mathrm{N}$ category $(\mathrm{p}<.001)$, lymph node metastasis ( $\mathrm{p}=.002)$, and HLA-I expression ( $\mathrm{p}=.029)$ (Fig. $3 A$ ) were associated with DSS. The presence of Tregs and immunologic group by HLA/Treg status were not associated with DSS ( $\mathrm{p}=.102$ and $\mathrm{p}=.080$, respectively). In the multivariate analysis, only distant metastasis $(\mathrm{p}=.032)$ was an independent prognostic factor for DSS (Table 2). In the univariate analysis for DFS, lymphovascular invasion ( $\mathrm{p}=.034)$, distant metastasis $(\mathrm{p}<.001), \mathrm{T}$ category $(\mathrm{p}<.001), \mathrm{N}$ category $(\mathrm{p}<.001)$, and histologic grade $(\mathrm{p}=.003)$ were significantly associated among the clinicopathological features. In the multivariate analysis for DFS, distant metastasis ( $\mathrm{p}=.001), \mathrm{T}$ category $(\mathrm{p}=.003)$, and histologic grade $(\mathrm{p}=.015)$ remained as independent prognostic factors (Table 2). HLA-I ( $\mathrm{p}=.863$ ) (Fig. 3B), Treg ( $=.347$ ), and immunologic group by HLA/Treg $(\mathrm{p}=.695)$ were not associated with DFS.

\section{Prognostic significance of HLA-I expression and Treg infiltration in the advanced disease group}

Of the 277 patients with advanced disease (American Joint Committee on Cancer stage II-IV), 27 patients (9.7\%) died of breast cancer and 53 (19.1\%) experienced disease recurrence or progression during the follow-up period. Positive HLA-I expres- sion and Treg infiltration was observed in 84.5\% (234/277) and $74.7 \%$ (207/277) of the cases, respectively. Among the clinicopathological features, HLA-I expression $(\mathrm{p}=.007)$ (Fig. 3C), perineural invasion $(\mathrm{p}=.001)$, distant metastasis $(\mathrm{p}<.001), \mathrm{T}$ category ( $\mathrm{p}<.001)$, and $\mathrm{N}$ category $(\mathrm{p}<.001)$ were associated with DSS in the univariate analysis. Based on the multivariate analysis, patients with negative HLA-I expression ( $\mathrm{p}=.034)$, distant metastasis ( $\mathrm{p}=.026)$, and high $\mathrm{N}$ category ( $\mathrm{p}=.034)$ had worse DSS (Table 2). However, in the univariate analysis for DFS, perineural invasion $(\mathrm{p}=.005)$, distant metastasis $(\mathrm{p}<.001)$, $\mathrm{T}$ category $(\mathrm{p}<.001)$, and $\mathrm{N}$ category $(\mathrm{p}<.001)$ were the only features that were significantly associated with the survival (Table 2), and HLA-I showed no prognostic significance ( $\mathrm{p}=.506)$ (Fig. 3D).

\section{Prognostic significance of HLA-I expression and Treg infiltration in intrinsic subtypes}

We also performed subgroup analysis according to $\mathrm{HR}$ status and intrinsic subtypes. In the 155 patients with HR-negative tumors, 18.7\% (29/155) had no peritumor Treg infiltration. Univariate analysis showed that tumors with Treg infiltration had better DSS than tumors without Treg infiltration (mean survival duration, 158.6 months vs. 106.3 months, respectively; $\mathrm{p}=.044)$. Other clinicopathological features that were significantly associated with DSS were perineural invasion ( $\mathrm{p}=.001)$, distant metastasis $(\mathrm{p}<.001), \mathrm{T}$ category $(\mathrm{p}<.001)$, and $\mathrm{N}$ category $(\mathrm{p}=.032)$. HLA-I expression $(\mathrm{p}=.227)$ and the immunologic 

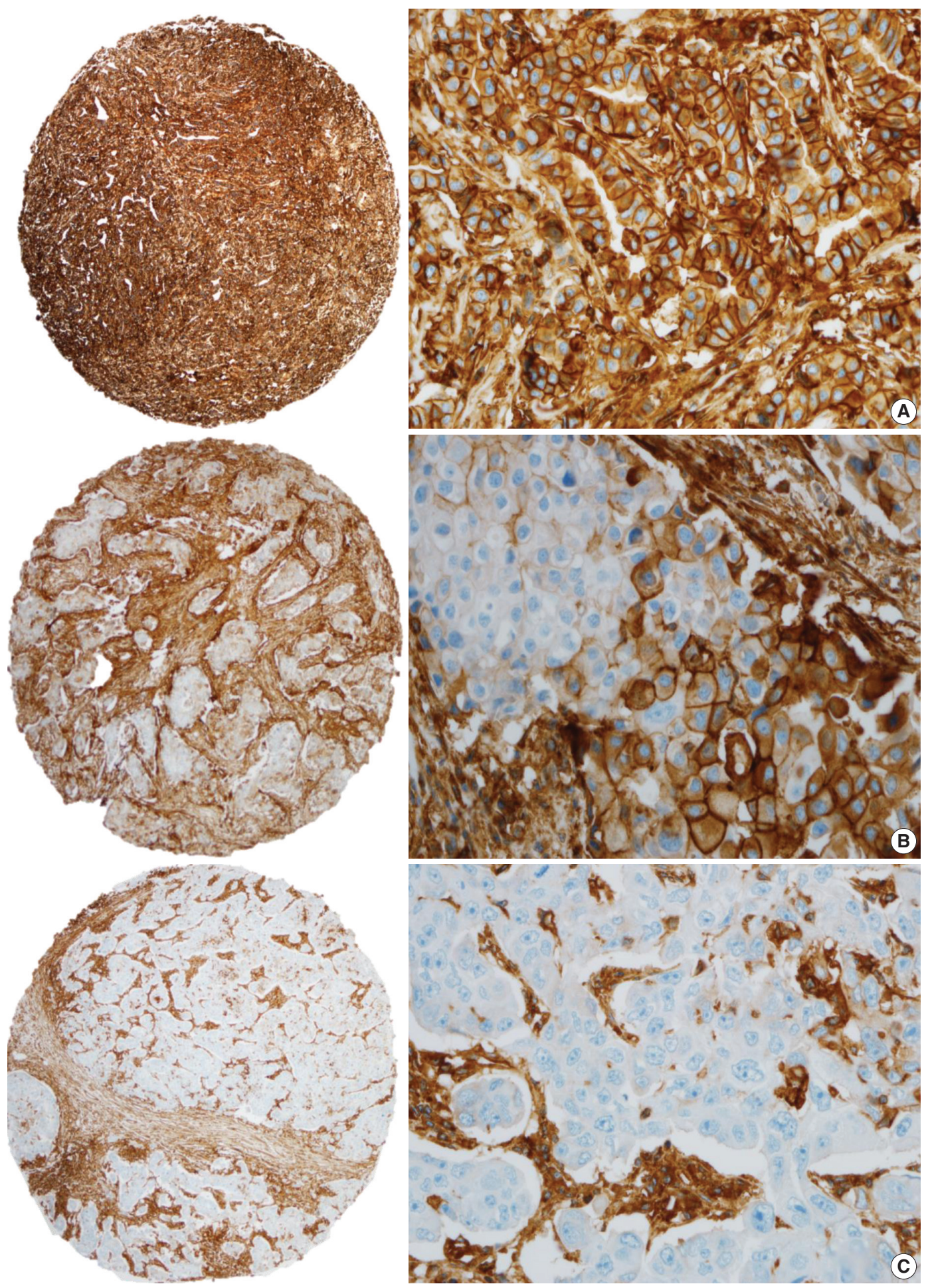

Fig. 1. Immunohistochemical staining of breast cancer tissues with anti-human leukocyte antigen class I (HLA-I) antibody. (A) Most of the HLA-I-positive cases showed a strong and diffuse membranous expression of HLA-I in breast cancer. In a HLA-I-positive case, the tumor cell membranes are completely stained with HLA-I. (B) A tumor with intermediate HLA-I expression (25\%-75\%) shows a heterogeneous staining pattern with a range of intensity. Partial loss of HLA-I expression in tumor cells is observed. (C) HLA-I-negative cases showed complete loss of HLA-I in breast cancer cells. Only the stromal cells are strongly stained and tumor cells are absent for HLA-I expression in cell membranes. 


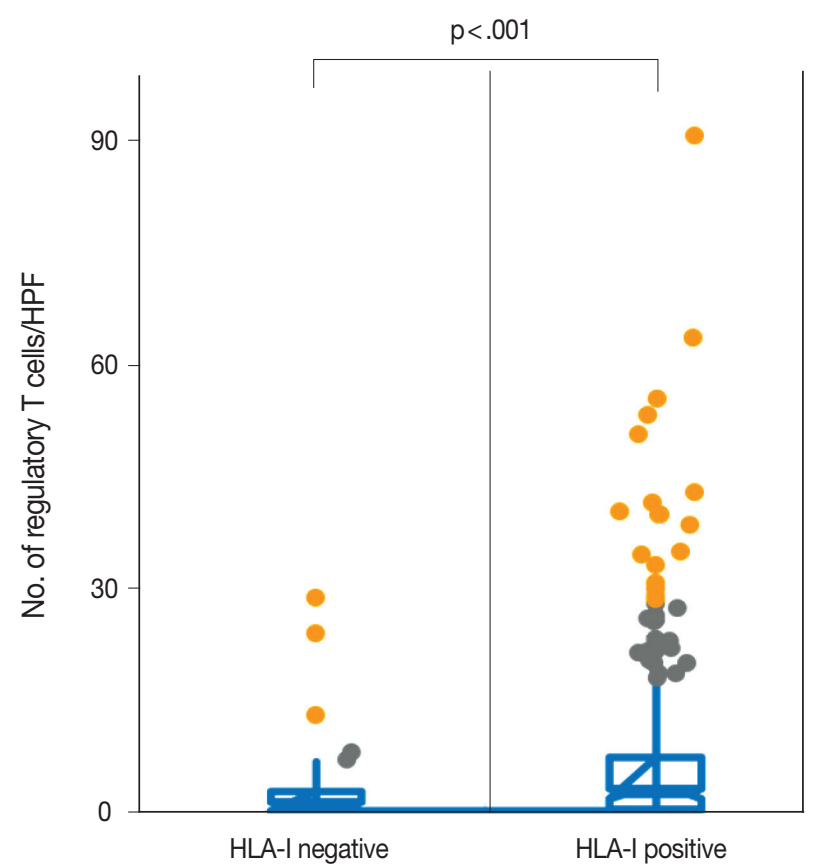

Fig. 2. The mean number of regulatory $T$ lymphocytes in the human leukocyte antigen class I (HLA-I)-negative and $\mathrm{H}\llcorner\mathrm{A}-\mathrm{I}-$ positive tumors was 2.1 cells/high-power field (HPF) and 6.3 cells/HPF, respectively. There was a statistically significant difference in the regulatory T cell infiltration between the two groups $(p<.001)$.

group by combination of HLA/Treg status $(\mathrm{p}=.080)$ were not associated with DSS in the HR-negative group. In the multivariate analysis, Treg infiltration had no statistically significant association with DSS after adjusting for other clinicopathological factors $(\mathrm{p}=.317)$. In univariate analysis for DFS, only nuclear grade $(\mathrm{p}=.010)$, lymphovascular invasion $(\mathrm{p}=.004)$, perineural invasion ( $\mathrm{p}<.001$ ), distant metastasis $(\mathrm{p}=.001)$, and $\mathrm{T}$ category $(\mathrm{p}<.001)$ were significantly associated with clinical outcome, but immunologic markers (e.g., HLA-I, Treg, immunologic group by combination of HLA/Treg status) had no prognostic significance.

When the HR-negative group was categorized into the HER2positive subtype and triple negative subtype, HLA-I status and Treg infiltration had no significant impact on clinical outcomes in both groups (all $\mathrm{p}>.05$ for DSS and DFS). The DSS of the patients with HER2-positive breast cancer based on HLA/Treg was not statistically evaluable due to non-occurrence of patient death during the study period in some subgroups. There was no difference between the HLA/Treg groups for other clinical outcomes in patients with the HER2-positive subtype and triple negative subtype (all $\mathrm{p}>.05$ ).

In the HR-positive group, Treg infiltration and HLA-I expression had no prognostic significance with respect to DSS and DFS (all $\mathrm{p}>.05$ ). In both luminal A and luminal B subtypes, Treg infiltration and HLA-I expression also had no prognostic significance with respect to DSS and DFS (all p > .05).

\section{DISCUSSION}

Several fundamental changes in cell physiology are considered hallmarks of cancer. One of these hallmarks is the ability of cancer cells to evade the host immune response. The host immune system can recognize and eliminate cells presenting abnormal tumor antigens, such as those against mutated oncoproteins. Altered HLA-I expression in tumor cells is one of the mechanisms that contributes to immune system evasion. ${ }^{3}$ The infiltration of Tregs is a host response to abnormal tumor antigens. ${ }^{16}$

In the present study, we found that $18.1 \%$ of the 465 primary breast cancer tissues showed total loss of HLA-I expression, which was correlated with decreased Treg infiltration. We found that a total loss of HLA-I expression was an unfavorable prognostic factor in the overall patient group as well as in the advanced disease group. A negative prognostic role for Tregs in breast cancer has been reported. ${ }^{17}$ However, Treg infiltration was not a significant prognostic factor in our study.

Little is known about the utility of the anti-pan HLA class I antibody EMR8-5 in breast cancer. EMR8-5 was first developed by a group led by Sato and can recognize denatured antigens in FFPE tissues. ${ }^{6}$ Most anti-HLA antibodies detect the heterodimeric structure of a $\beta$-microglobulin with a heavy chain or the native, allele-specific structure of HLA-I molecules. Unlike other anti-HLA-I antibodies (e.g., HC10 and HCA2) that show specificity to either HLA-A or -B/-C alleles, EMR8-5 reacts with all 17 types of HLA-A, $-\mathrm{B}$, and -C alleles in FFPE tissues. ${ }^{6}$ The conflicting results on the prognostic impact of HLA-I could arise from the different anti-HLA-I antibodies and varying specificity. ${ }^{5}$ In the present study, we provide compelling evidence using the EMR8-5 antibody that loss of HLA-I expression is a marker of poor prognosis in breast cancer patients. Patients with HLA-I-negative breast cancers had significantly worse prognosis compared with the patients with positive HLA-I expression ( $\mathrm{p}=$ .029) as determined by univariate analysis. Furthermore, HLAI itself was a significant prognostic factor for DSS without association of TNM factors in patients with advanced disease stages (stage II, III, and IV). Two of the four previous studies conducted with EMR8-5 demonstrated a significant association of HLA-I expression and DFS in breast cancer. ${ }^{910}$ However, after adjusting for other clinicopathological features in these studies, HLA-I was not a significant prognostic marker. Along with the previous results, our data suggest that the negative prognostic impact of 

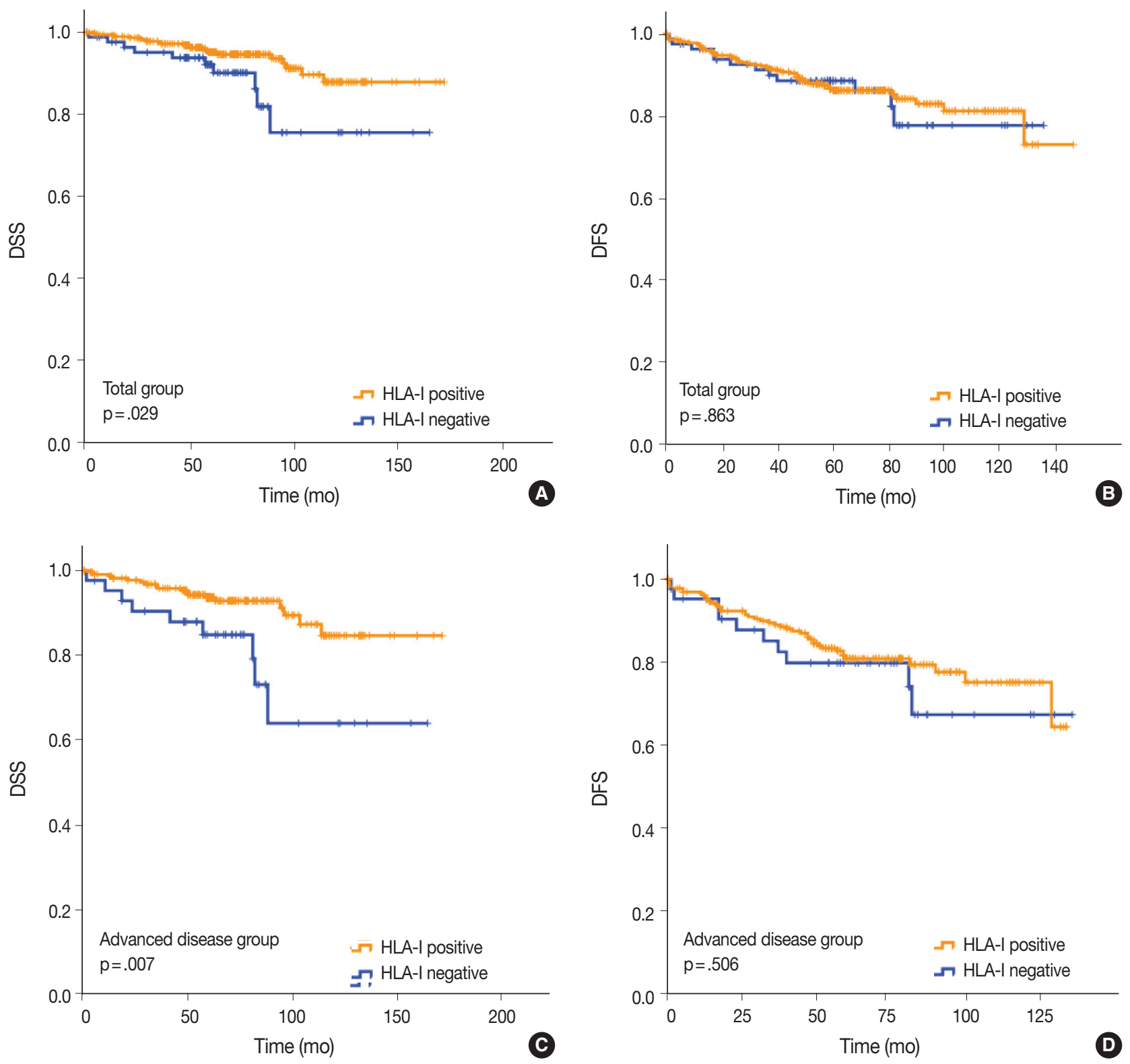

Fig. 3. (A) Disease-specific survival (DSS) of all patients with breast cancer based on human leukocyte antigen class I (HLA-I) expression ( $p=$ .029). (B) Disease-free survival (DFS) of all patients with breast cancer based on HLA-I expression ( $p=.863$ ). (C) DSS of the patient subgroup with stage II-IV breast cancer based on HLA-I expression $(p=.007)$. (D) DFS of the patient subgroup with stage II-IV breast cancer based on HLA-I expression $(p=.506)$.

HLA-I alteration may not be sufficient to alter the clinical course in the stage I group, which has good prognosis and with a 5-year relative survival rate of nearly $100 \%$. However, HLA-I expression was significantly associated with prognosis in the advanced stage group. ${ }^{10}$ The exact mechanism of HLA-I down-regulation and its association with prognosis is still unclear, and clarifying of the underlying mechanism in future studies may lead to novel therapeutic strategies for breast cancer.

In the current study, we also examined the relationship between
HLA-I expression and Treg infiltration, as this has not been explored. The number of tumor-infiltrating lymphocytes, although not specifically Tregs, was positively correlated with HLA expression in breast cancer. ${ }^{9}$ Immune-suppressing Tregs might consider HLA-I-positive tumors cells as normal cells and infiltrate to suppress immune attack, or tumor cells may influence Tregs to evade immune attack by effector $\mathrm{T}$ cells. ${ }^{8}$ In our study, the number of infiltrating Tregs was positively correlated with HLA-I expression in tumor cells. Regarding an association between loss of 
Table 2. Multivariate analysis for disease-specific survival and disease-free survival

\begin{tabular}{|c|c|c|c|c|c|c|c|c|c|c|c|c|}
\hline \multirow{3}{*}{ Variable } & \multicolumn{6}{|c|}{ Total patient group $(n=465)$} & \multicolumn{6}{|c|}{ Advanced breast cancer group (stage II, III, IV) ( $\mathrm{n}=277)$} \\
\hline & \multicolumn{3}{|c|}{ Disease-specific survival } & \multicolumn{3}{|c|}{ Disease-free survival } & \multicolumn{3}{|c|}{ Disease-specific survival } & \multicolumn{3}{|c|}{ Disease-free survival } \\
\hline & $\begin{array}{l}\text { Relative } \\
\text { risk }\end{array}$ & $\begin{array}{l}\text { Hazard ratio } \\
(95 \% \mathrm{Cl})\end{array}$ & $\begin{array}{c}p- \\
\text { value }\end{array}$ & $\begin{array}{l}\text { Relative } \\
\text { risk }\end{array}$ & $\begin{array}{l}\text { Hazard ratio } \\
(95 \% \mathrm{Cl})\end{array}$ & $\begin{array}{l}\mathrm{p}- \\
\text { value }\end{array}$ & $\begin{array}{l}\text { Relative } \\
\text { risk }\end{array}$ & $\begin{array}{c}\text { Hazard ratio } \\
(95 \% \mathrm{Cl})\end{array}$ & $\begin{array}{l}p-R \\
\text { value }\end{array}$ & $\begin{array}{l}\text { Relative } \\
\text { risk }\end{array}$ & $\begin{array}{l}\text { Hazard ratio } \\
(95 \% \mathrm{Cl})\end{array}$ & $\begin{array}{l}p- \\
\text { value }\end{array}$ \\
\hline HLA-I expression & 0.472 & $0.216-1.030$ & .059 & - & - & - & 0.407 & $0.177-0.936$ & .034 & - & - & - \\
\hline Lymphovascular invasion & 0.999 & $0.371-2.688$ & .998 & 0.760 & $0.386-1.495$ & .426 & - & - & - & - & - & - \\
\hline Perineural invasion & 1.518 & $0.622-3.704$ & .359 & 1.345 & $0.663-2.729$ & .412 & 1.682 & $0.664-4.266$ & .273 & 1.572 & $0.751-3.288$ & .230 \\
\hline Distant metastasis (M1) & 3.847 & $1.123-13.179$ & .032 & 5.027 & $1.928-13.103$ & .001 & 3.884 & $1.177-12.822$ & .026 & 4.586 & $1.784-11.786$ & .002 \\
\hline T category (reference 1) & & & .067 & & & .003 & & & .163 & & & .045 \\
\hline 2 & 1.591 & $0.667-3.794$ & .295 & 1.820 & 1.013-3.269 & .045 & 0.931 & $0.328-2.646$ & .894 & 1.053 & $0.487-2.276$ & .896 \\
\hline 3 & 2.174 & $0.566-8.358$ & .258 & 1.328 & $0.395-4.470$ & .646 & 1.303 & $0.310-5.471$ & .718 & 0.873 & $0.241-3.158$ & .835 \\
\hline 4 & 28.399 & $2.330-346.135$ & .009 & 76.642 & $6.085-965.396$ & .001 & 15.085 & $1.209-188.252$ & .0353 & 35.171 & $2.677-462.078$ & .007 \\
\hline $\mathrm{N}$ category (reference 0 ) & & & .056 & & & .115 & & & .034 & & & .210 \\
\hline 1 & 1.037 & $0.346-3.107$ & .949 & 1.189 & $0.600-2.357$ & .619 & 0.780 & $0.243-2.501$ & .677 & 0.700 & $0.340-1.439$ & .331 \\
\hline 2 & 3.262 & $1.081-9.848$ & .036 & 2.391 & $1.037-5.513$ & .041 & 2.628 & $0.924-7.476$ & .070 & 1.417 & $0.638-3.148$ & .392 \\
\hline 3 & 5.531 & $1.276-23.974$ & .022 & 3.098 & $0.968-9.917$ & .057 & 4.331 & $1.262-14.867$ & .020 & 2.139 & $0.776-5.892$ & .141 \\
\hline $\begin{array}{l}\text { Histologic grade } \\
\text { (grade } 1 \text { vs } 2,3 \text { ) }\end{array}$ & - & - & - & 2.753 & $1.219-6.218$ & .015 & - & - & - & - & - & - \\
\hline
\end{tabular}

Values of $p<.05$ in the univariate analysis were included in a multivariate analysis.

$\mathrm{Cl}$, confidence interval; HLA-I, human leukocyte antigen class I.

HLA-I expression and lower number of Tregs, HLA-I-negative tumors may have a lower density of tumor-infiltrating lymphocytes than HLA-I-positive tumors, because they are usually lowgrade, luminal A tumors. Our finding on the correlation between HLA-I expression and Tregs is in agreement with results of previous studies in breast cancer ${ }^{12}$ and gastric cancer. ${ }^{14}$ Further study is needed to reveal the underlying mechanism of the Treg and HLA-I interaction in cancers.

Total loss of HLA-I expression was less frequent in the metastatic tumors than in the primary tumors in our study $(18.1 \%$ vs $11 \%)$. Although this difference was not statistically significant, it may contradict our hypothesis that metastatic tumors would lose HLA-I expression during cancer progression. Limited studies have been conducted on the sequential development of metastases over time. In addition, it is not clear how HLA-I loss variants are generated in primary tumors and how they correlate with metastatic capacity. Previous studies on breast cancer ${ }^{20}$ and osteosarcoma ${ }^{21}$ suggested that metastases may not selectively originate from a subclone of tumor cells that acquired HLA loss in the primary site ("acquired" phenotype), but rather that tumors with inherent defects in HLA expression may have an advantage in metastasizing ("inherent" phenotype), which ultimately leads to poor survival. Our data also support the inherent phenotype hypothesis, but the small number of metastatic tumors and heterogeneous nature of cancer should be considered when interpreting this data. Further prospective investigations are needed to examine this hypothesis.
Some evidence has indicated that the presence of Tregs in the tumor microenvironment is an adverse prognostic marker in cancer. ${ }^{14,16}$ Considering the immunological nature of the interaction between HLA-I expression and Treg status, we hypothesized that the combination of these two markers would more accurately stratify prognostic groups. Our data, however, showed that Treg status had no independent prognostic value in the total group and subgroup analyses. A better understanding of the biological characteristics of Tregs in different tumor microenvironments is required to maximize their potential utility in tumor immunotherapy and to not oversimplify their role.

Recently, deleterious mutations in the gene encoding $\beta$-2microglobulin were found in melanoma and lung cancer tissues with acquired resistance to programmed death-ligand 1 inhibitors. Based on these findings, defects in the antigen presentation pathway were suggested as a mechanism of resistance to immune checkpoint inhibitors. ${ }^{22,23}$ This suggests the possibility that the expression and functional integrity of HLA antigens and related molecules in breast cancer could be a critical measure by which to choose candidate patients for immune checkpoint inhibitors in the future. Furthermore, restoring HLA expression could possibly prevent resistance to immunotherapy. ${ }^{24}$ Continuing research on this matter may be beneficial to advanced cancer patients.

This study has several limitations. Although we analyzed a large cohort, our study has the potential for selection bias because it is a retrospective study. Moreover, heterogeneity of HLA-I expression within each tumor may reduce the reliability of assess- 
ment results. Additional studies with whole tissue sections and a prospective design will be helpful in verifying the prognostic role of HLA-I in breast cancer.

In conclusion, we showed that the status of HLA-I expression is closely related to Treg infiltration and affects breast cancer patient survival, especially in patients with advanced disease. In the era of immunotherapy, HLA-I may be a promising prognostic marker and enable the application of more effective and precise immunotherapies for patients with advanced breast cancer.

\section{ORCID}

Hong Sik Park: https://orcid.org/0000-0003-0888-0711

Uiju Cho: https://orcid.org/0000-0002-6229-8418

So Young Im: https://orcid.org/0000-0002-5712-9003

Chang Young Yoo: https://orcid.org/0000-0001-7225-9896

Ji Han Jung: https://orcid.org/0000-0003-4191-4055

Young Jin Suh: https://orcid.org/0000-0001-8281-198X

Hyun Joo Choi: https://orcid.org/0000-0003-2292-424X

\section{Author Contributions}

Conceptualization: HJC.

Data curation: HSP, UC, HJC.

Formal analysis: HSP, UC, HJC.

Funding acquisition: HJC.

Investigation: HSP, UC, HJC.

Methodology: HSP, UC, SYI, CYY, JHJ, HJC.

Project administration: HJC.

Resources: HJC.

Supervision: HJC.

Validation: SYI, CYY, JHJ, YJS, HJC.

Visualization: HSP, UC, HJC.

Writing—original draft: HSP, UC, HJC.

Writing—review \& editing: HSP, UC, HJC.

\section{Conflicts of Interest}

The authors declare that they have no potential conflicts of interest.

\section{Acknowledgments}

This study was funded by the St. Vincent's Hospital Research Institute of Medical Science Foundation (SVHR-2016-13).

\section{REFERENCES}

1. Hanahan D, Weinberg RA. Hallmarks of cancer: the next generation.
Cell 2011; 144: 646-74.

2. Aptsiauri N, Cabrera T, Garcia-Lora A, Lopez-Nevot MA, RuizCabello F, Garrido F. MHC class I antigens and immune surveillance in transformed cells. Int Rev Cytol 2007; 256: 139-89.

3. Hicklin DJ, Marincola FM, Ferrone S. HLA class I antigen downregulation in human cancers: T-cell immunotherapy revives an old story. Mol Med Today 1999; 5: 178-86.

4. Garrido F, Ruiz-Cabello F, Cabrera T, et al. Implications for immunosurveillance of altered HLA class I phenotypes in human tumours. Immunol Today 1997; 18: 89-95.

5. Madjd Z, Spendlove I, Pinder SE, Ellis IO, Durrant LG. Total loss of MHC class I is an independent indicator of good prognosis in breast cancer. Int J Cancer 2005; 117: 248-55.

6. Torigoe T, Asanuma H, Nakazawa E, et al. Establishment of a monoclonal anti-pan HLA class I antibody suitable for immunostaining of formalin-fixed tissue: unusually high frequency of down-regulation in breast cancer tissues. Pathol Int 2012; 62: 303-8.

7. Chang CC, Campoli M, Restifo NP, Wang X, Ferrone S. Immune selection of hot-spot beta 2-microglobulin gene mutations, HLA-A2 allospecificity loss, and antigen-processing machinery component down-regulation in melanoma cells derived from recurrent metastases following immunotherapy. J Immunol 2005; 174: 1462-71.

8. de Kruijf EM, van Nes JG, Sajet A, et al. The predictive value of HLA class I tumor cell expression and presence of intratumoral Tregs for chemotherapy in patients with early breast cancer. Clin Cancer Res 2010; 16: 1272-80.

9. Lee HJ, Song IH, Park IA, et al. Differential expression of major histocompatibility complex class I in subtypes of breast cancer is associated with estrogen receptor and interferon signaling. Oncotarget 2016; 7: 30119-32.

10. Kaneko K, Ishigami S, Kijima Y, et al. Clinical implication of HLA class I expression in breast cancer. BMC Cancer 2011; 11: 454.

11. Lee HJ, Kim JY, Park IA, et al. Prognostic significance of tumor-infiltrating lymphocytes and the tertiary lymphoid structures in HER2positive breast cancer treated with adjuvant trastuzumab. Am J Clin Pathol 2015; 144: 278-88.

12. Chung YR, Kim HJ, Jang MH, Park SY. Prognostic value of tumor infiltrating lymphocyte subsets in breast cancer depends on hormone receptor status. Breast Cancer Res Treat 2017; 161: 409-20.

13. Vinay DS, Ryan EP, Pawelec G, et al. Immune evasion in cancer: mechanistic basis and therapeutic strategies. Semin Cancer Biol 2015; 35 Suppl: S185-98.

14. Ishigami S, Arigami T, Uenosono Y, et al. Cancerous HLA class I expression and regulatory $\mathrm{T}$ cell infiltration in gastric cancer. Cancer Immunol Immunother 2012; 61: 1663-9.

15. Curiel TJ, Coukos G, Zou L, et al. Specific recruitment of regulatory 
$\mathrm{T}$ cells in ovarian carcinoma fosters immune privilege and predicts reduced survival. Nat Med 2004; 10: 942-9.

16. deLeeuw RJ, Kost SE, Kakal JA, Nelson BH. The prognostic value of FoxP3+ tumor-infiltrating lymphocytes in cancer: a critical review of the literature. Clin Cancer Res 2012; 18: 3022-9.

17. Bates GJ, Fox SB, Han C, et al. Quantification of regulatory T cells enables the identification of high-risk breast cancer patients and those at risk of late relapse. J Clin Oncol 2006; 24: 5373-80.

18. Edge SB, Byrd DR, Compton CC, Fritz AG, Greene FL, Trotti A. AJCC cancer staging manual. 7th ed. New York: Springer, 2010.

19. Allred DC, Harvey JM, Berardo M, Clark GM. Prognostic and predictive factors in breast cancer by immunohistochemical analysis. Mod Pathol 1998; 11: 155-68.

20. Liu Y, Komohara Y, Domenick N, et al. Expression of antigen processing and presenting molecules in brain metastasis of breast cancer.
Cancer Immunol Immunother 2012; 61: 789-801.

21. Tsukahara T, Kawaguchi S, Torigoe T, et al. Prognostic significance of HLA class I expression in osteosarcoma defined by anti-pan HLA class I monoclonal antibody, EMR8-5. Cancer Sci 2006; 97: 1374-80.

22. Gettinger S, Choi J, Hastings K, et al. Impaired HLA class I antigen processing and presentation as a mechanism of acquired resistance to immune checkpoint inhibitors in lung cancer. Cancer Discov 2017; 7: 1420-35.

23. Zaretsky JM, Garcia-Diaz A, Shin DS, et al. Mutations associated with acquired resistance to PD-1 blockade in melanoma. N Engl J Med 2016; 375: 819-29.

24. Del Campo AB, Carretero J, Muñoz JA, et al. Adenovirus expressing beta2-microglobulin recovers HLA class I expression and antitumor immunity by increasing T-cell recognition. Cancer Gene Ther 2014; 21: 317-32. 